\title{
El desarrollo de habilidades investigativas: un eje en el proceso de formación de licenciados en educación
}

\section{Research skills development -an axis in teacher training process}

Ana Lucía Begué Lema ${ }^{1}$

\section{Resumen}

El tema del presente escrito está articulado por dos aspectos fundamentales: la formación de licenciados y la formación para la investigación, aspectos que constituyen una unidad, puesto que el tipo de formación que se reciba en la institución de educación superior se debe ver reflejado en un ejercicio profesional caracterizado por un pensamiento reflexivo, crítico, autónomo, lógico y flexible, que permita responder a las necesidades del entorno y de los estudiantes. En este sentido se presenta la manera en que se articula la formación para la investigación en las Licenciaturas en Educación del tecnológico de Antioquia-Institución Universitaria.

Palabras clave: Formación, habilidades investigativas, modelo pedagógico

\section{Abstract}

The topic this paper addresses is articulated by two basic aspects: teacher formation and research training. Both make up a unity, since formation at college must be reflected in a professional practice characterized by reflexive, critical, autonomous, logical, and flexible thinking, which allows meeting the needs from the

Texto logrado con el apoyo de los estudiantes de Licenciatura en Educacion Básica con énfasis en Humanidades y Lengua Castellana (LEBHLC): Stephanie Pérez Ortiz, y Santiago Alzate Hincapié, en las fases de planeación y elaboración de borrador o pre-escritura. 
environment and students. This is the purpose for showing how research training is delivered in Teaching Programas at Tecnológico de Antioquia College.

Keywords: Formation, research skills, pedagogical model.

La preocupación por la formación de educadores en Colombia data de hace varias décadas. De una formación tecnológica se pasó a finales de los años 70 , a un nivel de formación de licenciatura de cuatro ańos y en el 2000, a partir del decreto 272 de 1998, a licenciaturas de cinco años, cuya fundamentación se enfocó en la pedagogía, en la didáctica y en la formación investigativa a partir de prácticas reflexivas que, a su vez, generaran un cambio social acorde con el contexto local, regional y nacional.

Aunque el decreto 272 fue derogado por el 2566 de 2003, los programas de licenciatura se reestructuraron a partir de los lineamientos del 272. La formación de educadores se transformó al pasar de una visión centrada en una racionalidad técnica (Schon, 1992) en la cual el profesor debía resolver problemas instrumentales a partir de teorías y técnicas derivadas de un conocimiento científico, a una visión fundamentada en la racionalidad práctica caracterizada por el uso de teorías que se ponen en función de los problemas prácticos, según las finalidades y medios requeridos para solucionarlos. Esta nueva visión se caracteriza por la capacidad que deben tener los maestros para reflexionar no sólo sobre las propias prácticas, sino también sobre las que circulan en los espacios cotidianos de las instituciones educativas.

Esta transformación en el proceso de formación de educadores trajo como consecuencia una reflexión sobre los aspectos a considerar desde lo pedagógico, lo personal y las exigencias relacionadas con los saberes generales y específicos a manejar por los estudiantes en los programas de licenciaturas. (Ministerio de Educación Nacional, 1996, idea orientadora No 82).

Desde lo pedagógico se sugiere desarrollar una fundamentación epistemológica de la pedagogía como mediadora cultural, que se evidencie en la capacidad de generar prácticas autónomas a partir de una mirada reflexiva de las mismas. En el ámbito de ser persona, la exigencia se encamina a educadores éticos, sensibles, trascendentes y con identidad; por último, los saberes requeridos son el pedagógico, el cultural, el interdisciplinario, el investigativo, el reflexivo, además de la capacidad para integrar la información en un contexto sociohistórico y político.

Las anteriores consideraciones se vuelven operativas en el proceso de formación, en buena medida, a través del modelo pedagógico que se implemente en la institución de educación superior, entendido como "un constructor teórico y de interacción en un contexto específico que alienta una perspectiva futura de formación y que se construye para concretar propósitos e intencionalidades referidas a un proyecto de sociedad, de cultura y de educación" (Ministerio de Educación Nacional, 1996, idea orientadora No 22).

Desde esta perspectiva, el modelo debe permitir movilizar prácticas pedagógicas que respondan a las necesidades del medio y que trasciendan la imitación de concepciones enciclopédicas basadas en la memorización y transmisión mecánica del conocimiento, es decir, prácticas 
que aumenten la capacidad de vivir en medio de la incertidumbre, para transformarse y provocar el cambio; o como lo expresa Francisco Imbernon (1996. p.32) docente investigador de la Universidad de Salamanca "el profesor o la profesora ya no es un técnico que desarrolla o implementa innovaciones prescritas, sino que es un profesional que debe participar activa y críticamente, desde y en su propio contexto, en un proceso dinámico, flexible, en el verdadero proceso de innovación"

En este marco contextual, el Tecnológico de Antioquia asume un modelo pedagógico socio crítico, caracterizado por entender que el ser humano es quien construye el conocimiento a partir de la interacción con la cultura y con los objetos e instrumentos que ofrece el contexto, en torno al desarrollo de la autonomía, la creatividad, la afectividad, la participación colectiva y la sensibilidad social, favoreciendo así la adaptación del individuo al entorno y la transformación del mismo a partir de la prevención y resolución de problemas.

En el modelo, uno de los ejes a tener en cuenta para la formación de maestros con dichas características, es el relacionado con el desarrollo de habilidades investigativas, dentro de un marco de investigación pedagógica que Bernardo Gómez Restrepo (1999), entiende como aquel que abarca toda investigación relacionada con el área, a partir de la descripción, clasificación, explicación, experimentación y control de los factores objeto de estudio, dando lugar al desarrollo de procesos ajustados a los contextos.

En este orden de ideas, María Guadalupe Moreno (2005), entiende la formación para la investigación como un proceso que implica prácticas y actores diversos, y que se concreta en un quehacer académico consistente en promover y facilitar, preferentemente de manera sistematizada, el acceso a los conocimientos, el desarrollo de habilidades, hábitos y actitudes, y la internalización de valores, que demanda la realización de la práctica denominada investigación. Este proceso comienza con el desarrollo de ciertas habilidades que posteriormente contribuirán de manera fundamental a la realización de investigaciones de buena calidad.

La propuesta de Moreno sobre las habilidades investigativas a desarrollar en los estudiantes, se puede asumir para la investigación en el aula que deben desarrollar los educadores en formación y posteriormente en su ejercicio profesional, como un proceso de construcción de conocimiento que implica la intervención, en forma continua pero diferenciada, de procesos de orden cognitivo relacionados con la acción y el efecto de conocer. Estos se sintetizan en el siguiente cuadro: 


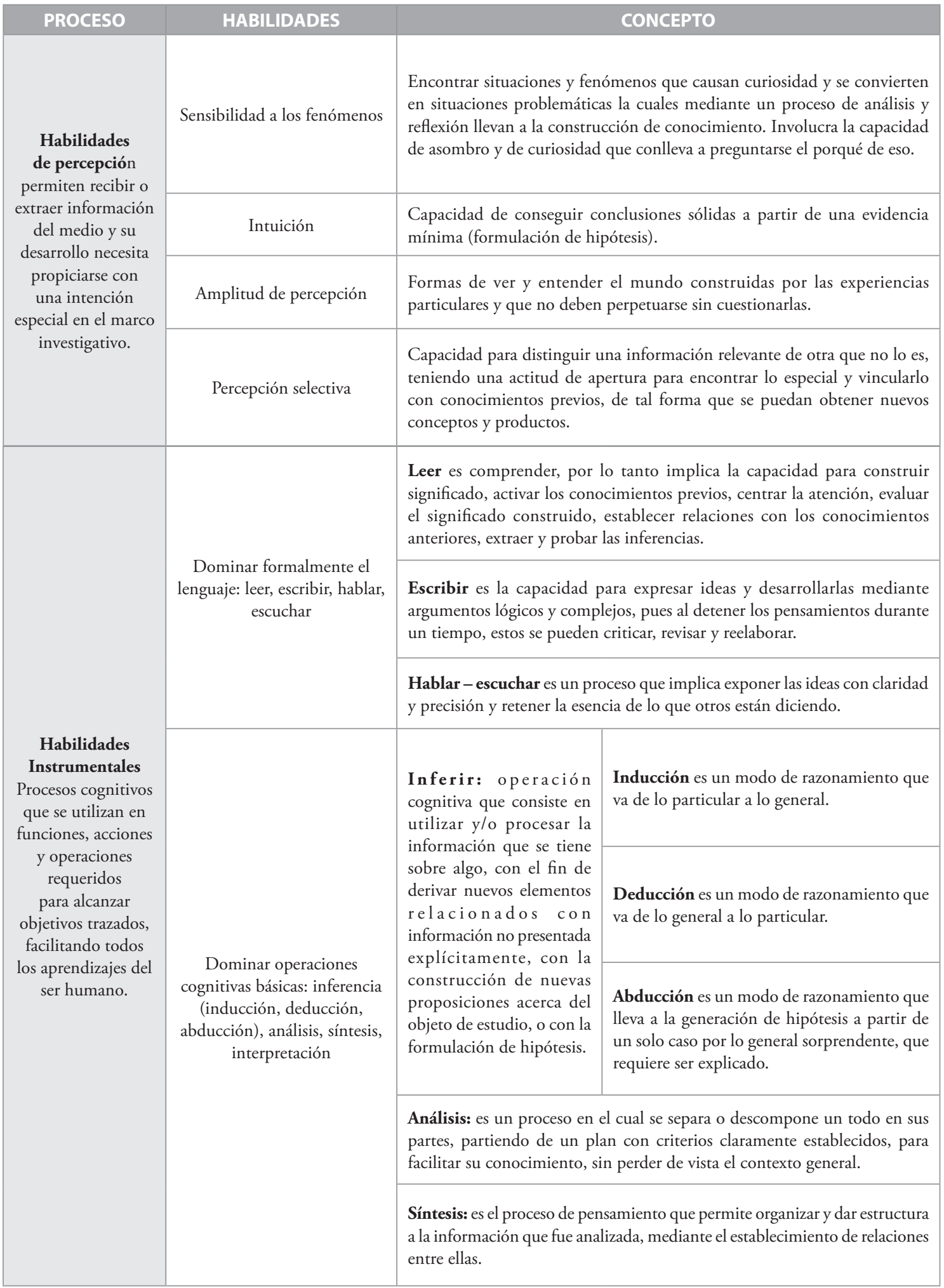




\begin{tabular}{|c|c|c|}
\hline PROCESO & HABILIDADES & CONCEPTO \\
\hline & & $\begin{array}{l}\text { Interpretación: los procesos de análisis y síntesis arrojan nuevos elementos } \\
\text { que permiten establecer relaciones, construir explicaciones, "añadir sentido, } \\
\text { leer entre líneas, llenar claros y extender un material dado dentro de los } \\
\text { límites de ese material" (Raths, 1988, p 257). En otras palabras, interpretar } \\
\text { es descubrir o construir un dignificado para lo encontrado al realizar el } \\
\text { examen de datos o información. }\end{array}$ \\
\hline & Saber observar & $\begin{array}{l}\text { Capacidad perceptual intencionada que se lleva a cabo con apoyo de los } \\
\text { cinco sentidos; supone ir más allá de lo que se percibe y su fin primordial } \\
\text { es recoger información acerca de un objeto de interés en el cual se centra } \\
\text { la atención (hechos, fenómenos, actitudes, conductas, comportamientos, } \\
\text { interacciones, procesos). } \\
\text { La observación está permeada por los elementos lingüísticos y culturales } \\
\text { del observador y en ella entran operaciones cognitivas como el análisis, la } \\
\text { síntesis, identificación, discriminación, comparación y jerarquización de } \\
\text { lo observado, con apoyo de una perspectiva teórica previamente elegida. }\end{array}$ \\
\hline & Saber Preguntar & $\begin{array}{l}\text { Preguntar es la mediación por excelencia para acceder al conocimiento, } \\
\text { pues todo conocimiento comienza por una pregunta, por la curiosidad. La } \\
\text { habilidad de preguntar hace referencia al tipo de pregunta que lleva implícito } \\
\text { el deseo de conocer, de indagar, de explicarse lo que de momento no parece } \\
\text { tener respuesta y de cuestionar lo establecido o poner en duda lo que se ha } \\
\text { tenido como verdadero. }\end{array}$ \\
\hline \multirow{5}{*}{$\begin{array}{l}\text { Habilidades de } \\
\text { pensamiento } \\
\text { Modos de pensar } \\
\text { que permiten la } \\
\text { realización de tareas } \\
\text { complejas. }\end{array}$} & Pensar críticamente & $\begin{array}{l}\text { La habilidad para pensar de una manera crítica implica la capacidad para } \\
\text { juzgar la credibilidad de determinadas afirmaciones, sopesar pruebas, valorar } \\
\text { la solidez lógica de distintas deducciones, discernir argumentos en contra, } \\
\text { discernir hipótesis alternativas. }\end{array}$ \\
\hline & Pensar lógicamente & $\begin{array}{l}\text { La habilidad para pensar de una manera lógica implica la capacidad para } \\
\text { dar orden y sentido a las ideas, expresarlas y apoyarlas coherentemente, } \\
\text { establecer inferencias válidas, construir argumentos consistentes, detectar } \\
\text { incongruencias presentes en las investigaciones de otros y de sí mismo, } \\
\text { encontrar y/o generar el sentido de una estructura explicativa, articular } \\
\text { de manera congruente los quehaceres y los productos de la investigación }\end{array}$ \\
\hline & Pensar reflexivamente & $\begin{array}{l}\text { La habilidad para pensar de una manera reflexiva implica la capacidad para } \\
\text { realizar un examen exhaustivo de todos los elementos que pueden orientar } \\
\text { en su momento, la emisión de un juicio coherentemente sustentado. Tiene } \\
\text { como base los conocimientos previos que la persona ha construido, puestos } \\
\text { en contraste con los nuevos elementos que va encontrando, lo que modifica } \\
\text { los esquemas conceptuales existentes. }\end{array}$ \\
\hline & Pensar de manera autónoma & $\begin{array}{l}\text { La habilidad para pensar de una manera independiente implica la capacidad } \\
\text { para intervenir en la construcción de ideas, de soltarse, de exponerse al } \\
\text { error, de asumirse como ser que puede criticar y generar sus propias ideas, } \\
\text { discernir con que base asume o critica a las de los demás, definiendo su } \\
\text { personalidad intelectual. }\end{array}$ \\
\hline & Flexibilizar el pensamiento & $\begin{array}{l}\text { Implica tener una visión dinámica del problema de investigación, puesto } \\
\text { que la pregunta en que se concreta un problema, está en espera de una } \\
\text { respuesta que siempre es movible y que requiere de la incorporación de } \\
\text { nuevos elementos. }\end{array}$ \\
\hline
\end{tabular}




\begin{tabular}{|c|c|c|}
\hline PROCESO & HABILIDADES & CONCEPTO \\
\hline \multirow{6}{*}{$\begin{array}{l}\text { Habilidades de } \\
\text { construcción } \\
\text { conceptual } \\
\text { Habilidades cuyo } \\
\text { desarrollo posibilita } \\
\text { desempeńos } \\
\text { vinculados con } \\
\text { el proceso de } \\
\text { investigación, } \\
\text { desde el aspecto } \\
\text { conceptual }\end{array}$} & $\begin{array}{l}\text { Apropiarse y reconstruir las } \\
\text { ideas de otros }\end{array}$ & $\begin{array}{l}\text { Implica comprender claramente el contenido de las ideas de los otros, hasta } \\
\text { el punto de expresarlas de una manera diferente, sin cambiar el sentido de } \\
\text { las ideas en cuestión y precisando que se están utilizando ideas que son } \\
\text { originales de otros autores (parafrasear) }\end{array}$ \\
\hline & Generar ideas & $\begin{array}{l}\text { Capacidad que hace referencia al trabajo intelectual cuyo producto se } \\
\text { concreta en la construcción y planteamiento de ideas acerca de un objeto de } \\
\text { estudio, las cuales no han sido presentadas por otros, o bien, son presentadas } \\
\text { por alguien sin conocer que dichas ideas ya fueron trabajadas antes. }\end{array}$ \\
\hline & $\begin{array}{l}\text { Organizar lógicamente, } \\
\text { exponer y defender ideas }\end{array}$ & $\begin{array}{l}\text { Capacidad para comunicar y defender las ideas, que se apoya por la habilidad } \\
\text { para pensar lógicamente y por las relacionadas con el dominio formal } \\
\text { del lenguaje. Implica la capacidad de argumentar, es decir de presentar } \\
\text { razones suficientes y desarrolladas que puedan sustentar un planteamiento } \\
\text { determinado. }\end{array}$ \\
\hline & Problematizar & $\begin{array}{l}\text { Proceso que parte de la capacidad de formular preguntas y supone el } \\
\text { acercamiento a los fenómenos en forma similar a la función del zoom, es } \\
\text { decir, mirar con diversos niveles de distanciamiento y de tamaño las zonas } \\
\text { de interés, con el fin de percibir aquello que no es fácilmente perceptible, } \\
\text { de tal forma que se facilite la comprensión de la situación. }\end{array}$ \\
\hline & Construir objetos de estudio & $\begin{array}{l}\text { Construcción a partir de la cual es posible contar con elementos para } \\
\text { plantear un problema de investigación, lo que supone análisis y reflexión de } \\
\text { las situaciones problémicas, identificación de conceptos considerados como } \\
\text { centrales en las preguntas iniciales, la ubicación de esos conceptos en redes } \\
\text { que permiten establecer relaciones entre ellos y otros, el establecimiento de } \\
\text { múltiples relaciones entre conceptos, la búsqueda de fuentes documentales, } \\
\text { la selección de la relación que se convertirá en objeto de estudio y finalmente, } \\
\text { construir los significados con los que se trabajarán los conceptos y las } \\
\text { relaciones. }\end{array}$ \\
\hline & $\begin{array}{l}\text { Realizar síntesis conceptuales } \\
\text { creativas }\end{array}$ & $\begin{array}{l}\text { Implica poner en contacto teorías de diversos autores, relacionando cosas } \\
\text { que no estaban relacionadas previamente. }\end{array}$ \\
\hline \multirow{5}{*}{$\begin{array}{l}\text { Habilidades de } \\
\text { construcción } \\
\text { metodológica } \\
\text { Habilidades } \\
\text { que permiten el } \\
\text { desarrollo metódico } \\
\text { del proceso de } \\
\text { investigación }\end{array}$} & $\begin{array}{l}\text { Construir el método de } \\
\text { investigación }\end{array}$ & $\begin{array}{l}\text { Supone plantear de manera anticipada y global la estrategia de acción, } \\
\text { reflexión, contraste de referentes teóricos y empíricos, articulación, etc. que } \\
\text { posibilite la construcción del conocimiento, para el caso de la investigación } \\
\text { a realizar. }\end{array}$ \\
\hline & $\begin{array}{l}\text { Hacer pertinente el método } \\
\text { de construcción del } \\
\text { conocimiento }\end{array}$ & $\begin{array}{l}\text { Implica reformular y adecuar la estrategia elegida para la construcción del } \\
\text { conocimiento. }\end{array}$ \\
\hline & Construir observables & $\begin{array}{l}\text { Capacidad para relacionar categorías conceptuales con referentes empíricos } \\
\text { que se han seleccionado como indicadores de las mismas, haciéndolas } \\
\text { observables. }\end{array}$ \\
\hline & $\begin{array}{l}\text { Diseñar instrumentos y } \\
\text { procedimientos de búsqueda, } \\
\text { recuperación y generación de } \\
\text { información }\end{array}$ & Capacidad para encontrar información útil y pertinente para el proceso. \\
\hline & $\begin{array}{l}\text { Manejar y diseñar técnicas } \\
\text { para la organización, } \\
\text { sistematización y análisis de } \\
\text { la información }\end{array}$ & $\begin{array}{l}\text { Capacidad para construir las técnicas pertinentes para la investigación y el } \\
\text { abordaje del objeto de estudio. }\end{array}$ \\
\hline
\end{tabular}




\begin{tabular}{|c|c|c|}
\hline PROCESO & HABILIDADES & CONCEPTO \\
\hline \multirow{4}{*}{$\begin{array}{l}\text { Habilidades de } \\
\text { construcción } \\
\text { social del } \\
\text { conocimiento } \\
\text { Destacan el carácter } \\
\text { eminentemente } \\
\text { social de los } \\
\text { procesos de } \\
\text { producción del } \\
\text { conocimiento y de } \\
\text { los productos de la } \\
\text { investigación }\end{array}$} & Trabajar en grupo & $\begin{array}{l}\text { Implica pertenecer a un círculo de personas con las que se establecen } \\
\text { vínculos, relaciones e interacciones. Las cuales se constituyen en referencia, } \\
\text { tanto para las necesidades individuales, como para los intereses colectivos, } \\
\text { implica trabajar con los otros en objetivos comunes compartiendo el trabajo } \\
\text { mismo, las indagaciones, las intenciones, las conceptualizaciones, los logros, } \\
\text { etc. Demanda capacidad de diálogo, tolerancia, suposición y apertura para } \\
\text { interactuar con los otros. }\end{array}$ \\
\hline & $\begin{array}{l}\text { Socializar el proceso } \\
\text { de construcción de } \\
\text { conocimiento }\end{array}$ & $\begin{array}{l}\text { Implica aprender a relacionarse con los otros en términos de los intereses de } \\
\text { investigación, de tal forma que la productividad de cada miembro se fusione } \\
\text { y se integre en la meta colectiva. }\end{array}$ \\
\hline & Socializar el conocimiento & $\begin{array}{l}\text { Capacidad para compartir, dar a conocer y difundir el conocimiento } \\
\text { construido. }\end{array}$ \\
\hline & Comunicar & $\begin{array}{l}\text { Implica el desarrollo del plano interpersonal, en el cual es importante } \\
\text { la competencia en el uso pragmático del lenguaje teniendo en cuenta los } \\
\text { propósitos y necesidades de la comunicación, el lugar, los interlocutores, los } \\
\text { roles asumidos por éstos. En el plano lingüístico implica el correcto dominio } \\
\text { formal del lenguaje, además del correcto dominio de las reglas gramaticales. } \\
\text { En el plano cognitivo, la comunicación requiere de la capacidad para pensar } \\
\text { lógicamente, es decir, para organizar las ideas, categorizarlas, jerarquizarlas y } \\
\text { argumentarlas, teniendo pleno dominio de lo que se comunica. }\end{array}$ \\
\hline \multirow{5}{*}{$\begin{array}{c}\text { Habilidades } \\
\text { metacognitivas } \\
\text { Hace referencia } \\
\text { a las habilidades } \\
\text { relacionadas } \\
\text { con el control y } \\
\text { evaluación del } \\
\text { conocimiento } \\
\text { producido en la } \\
\text { investigación, y de } \\
\text { la forma de acceder } \\
\text { al mismo }\end{array}$} & $\begin{array}{l}\text { Objetivar la involucración } \\
\text { personal con el objeto de } \\
\text { conocimiento }\end{array}$ & $\begin{array}{l}\text { Hace referencia a la necesidad de volver consciente la involucración afectiva } \\
\text { y cognitiva con el objeto de estudio, pasando de un estado ingenuo a uno } \\
\text { crítico. }\end{array}$ \\
\hline & $\begin{array}{l}\text { Autorregular los procesos } \\
\text { cognitivos }\end{array}$ & $\begin{array}{l}\text { Consiste en hacer intervenir, reorientar, o bien interrumpir ciertos procesos } \\
\text { cognitivos, en función de la dirección particular en la que se está avanzando } \\
\text { durante la producción del conocimiento }\end{array}$ \\
\hline & $\begin{array}{l}\text { Autocuestionar la pertinencia } \\
\text { de las acciones relacionadas } \\
\text { con la construcción del } \\
\text { conocimiento }\end{array}$ & $\begin{array}{l}\text { Capacidad para evaluar y controlar el curso y la pertinencia de las formas } \\
\text { de acercamiento al objeto de estudio y efectuar los cambios pertinentes se } \\
\text { acuerdo con las necesidades. }\end{array}$ \\
\hline & $\begin{array}{l}\text { Revalorar los acercamientos } \\
\text { al objeto de estudio }\end{array}$ & $\begin{array}{l}\text { Supone la capacidad para aceptar la existencia de diversos modos de } \\
\text { acercamiento al objeto de estudio lo que implica la necesidad de evaluar la } \\
\text { manera elegida para ajustarla si es necesario. }\end{array}$ \\
\hline & $\begin{array}{l}\text { Autoevaluar la consistencia } \\
\text { y la validez de los productos } \\
\text { generados }\end{array}$ & $\begin{array}{l}\text { Son las evaluaciones de los productos parciales y globales desde un sustento } \\
\text { epistemológico, su coherencia y congruencia en lo general y lo particular. } \\
\text { Da cuenta del porqué, el para que y el cómo de los procesos generados. }\end{array}$ \\
\hline
\end{tabular}

Cuadro No 1: Fuente: Ana Lucía Begué Lema

El desarrollo de estas habilidades se concreta, en las licenciaturas en educación del Tecnológico de Antioquia, a partir del proceso de investigación formativa articulado a las prácticas que realizan los estudiantes durante los diez (10) semestres académicos, propendiendo así por el mejoramiento continuo tanto de los estudiantes en práctica, como de los docentes y de las instituciones en donde se realizan.

El proceso se inicia en cada nivel con la formulación de una pregunta pedagógica 
o investigativa que los estudiantes deben responder mediante la aplicación de técnicas de investigación (la observación, la encuesta, la historia de vida, la entrevista, el grupo focal, el estudio de caso y la línea de base). Una vez aplicada la técnica deben registrar el proceso de la práctica y los resultados de la técnica en el anecdotario, en el diario pedagógico o en el diario de campo; sistematizar la información y hacer entrega de productos específicos en cada nivel, que según el caso serán: el informe descriptivo, el informe analítico, el informe interpretativo, el informe argumentativo, el ensayo, el artículo y la sistematización de las experiencias investigativas.

En el siguiente cuadro se sintetiza el proceso:

\begin{tabular}{|c|c|c|c|c|c|}
\hline \multirow{2}{*}{ PRÁCTICA } & \multicolumn{5}{|c|}{ FACTORES / PROCESOS } \\
\hline & INDAGACIÓN & HABILIDAD & TÉCNICA & INSTRUMENTO & PRODUCTO \\
\hline I & Pregunta pedagógica & $\begin{array}{l}\text { Descripción, } \\
\text { narración y análisis }\end{array}$ & Observación & $\begin{array}{l}\text { Guía de observación, } \\
\text { anecdotario y } \\
\text { protocolo }\end{array}$ & $\begin{array}{l}\text { Informe } \\
\text { descriptivo }\end{array}$ \\
\hline II & Pregunta pedagógica & $\begin{array}{l}\text { Descripción, análisis } \\
\text { e interpretación }\end{array}$ & Encuesta & $\begin{array}{l}\text { Cuestionario, } \\
\text { anecdotario, } \\
\text { protocolo y reseña }\end{array}$ & $\begin{array}{l}\text { Informe } \\
\text { analítico }\end{array}$ \\
\hline III & Pregunta pedagógica & $\begin{array}{l}\text { Descripción, análisis } \\
\text { e interpretación }\end{array}$ & $\begin{array}{l}\text { Entrevista y } \\
\text { grupo focal / } \\
\text { Historia de Vida }\end{array}$ & $\begin{array}{l}\text { Caracterización } \\
\text { del desarrollo, } \\
\text { anecdotario y reseña }\end{array}$ & $\begin{array}{l}\text { Informe } \\
\text { interpretativo }\end{array}$ \\
\hline IV & Pregunta pedagógica & $\begin{array}{l}\text { Descripción, análisis } \\
\text { e interpretación }\end{array}$ & $\begin{array}{l}\text { Entrevista y } \\
\text { grupo focal / } \\
\text { Historia de vida }\end{array}$ & $\begin{array}{l}\text { Proyecto de aula, } \\
\text { anecdotario y } \\
\text { relatoría }\end{array}$ & $\begin{array}{l}\text { Informe } \\
\text { interpretativo }\end{array}$ \\
\hline $\mathbf{V}$ & Pregunta pedagógica & $\begin{array}{l}\text { Descripción, análisis } \\
\text { e interpretación }\end{array}$ & $\begin{array}{l}\text { Historia de vida } \\
\text { / Estudio de } \\
\text { caso }\end{array}$ & $\begin{array}{l}\text { Proyecto de aula, } \\
\text { diario pedagógico y } \\
\text { relatoría }\end{array}$ & $\begin{array}{l}\text { Informe } \\
\text { argumentativo }\end{array}$ \\
\hline VI & Pregunta pedagógica & $\begin{array}{l}\text { Descripción, análisis } \\
\text { e interpretación }\end{array}$ & Estudio de caso & $\begin{array}{l}\text { Proyecto de aula y } \\
\text { diario pedagógico }\end{array}$ & $\begin{array}{l}\text { Informe } \\
\text { argumentativo / } \\
\text { ensayo }\end{array}$ \\
\hline VII & Pregunta pedagógica & $\begin{array}{l}\text { Descripción, análisis } \\
\text { e interpretación }\end{array}$ & Estudio de caso & $\begin{array}{l}\text { Proyecto de aula y } \\
\text { diario pedagógico }\end{array}$ & $\begin{array}{l}\text { Sistematización } \\
\text { de experiencias / } \\
\text { ensayo }\end{array}$ \\
\hline VIII & $\begin{array}{l}\text { Pregunta pedagógica e } \\
\text { investigativa }\end{array}$ & $\begin{array}{l}\text { Descripción, análisis, } \\
\text { interpretación y } \\
\text { categorización }\end{array}$ & Línea de base & $\begin{array}{l}\text { Proyecto de aula y } \\
\text { diario pedagógico }\end{array}$ & $\begin{array}{l}\text { Sistematización } \\
\text { de experiencias / } \\
\text { artículo }\end{array}$ \\
\hline IX & $\begin{array}{l}\text { Pregunta investigativa } \\
\text { articulada al objeto de } \\
\text { enseńanza }\end{array}$ & $\begin{array}{l}\text { Descripción, análisis, } \\
\text { interpretación y } \\
\text { categorización }\end{array}$ & $\begin{array}{l}\text { Fortalecimiento } \\
\text { de técnicas }\end{array}$ & $\begin{array}{l}\text { Proyecto investigativo } \\
\text { y diario de campo }\end{array}$ & $\begin{array}{l}\text { Artículo } \\
\text { y primera } \\
\text { socialización }\end{array}$ \\
\hline $\mathbf{X}$ & $\begin{array}{l}\text { Pregunta investigativa } \\
\text { articulada al objeto de } \\
\text { enseñanza }\end{array}$ & $\begin{array}{l}\text { Descripción, análisis, } \\
\text { interpretación y } \\
\text { categorización }\end{array}$ & $\begin{array}{l}\text { Fortalecimiento } \\
\text { de técnicas }\end{array}$ & $\begin{array}{l}\text { Proyecto investigativo } \\
\text { y diario de campo }\end{array}$ & $\begin{array}{l}\text { Artículo, } \\
\text { sistematización } \\
\text { del proyecto y } \\
\text { socialización de } \\
\text { resultados }\end{array}$ \\
\hline
\end{tabular}

Cuadro No 2: Fuente: Manual de prácticas interinstitucionales Tecnológico de Antioquia, 2008 
El desarrollo de habilidades investigativas: un eje en el proceso de formación de licenciados en educación TaleA

Los docentes, por su parte, deben sistematizar el desarrollo del proceso de investigación formativa y de las habilidades trabajadas, además de las diferentes respuestas a la pregunta que genera la indagación en cada práctica. De esta manera se pretende tener la evidencia del proceso y realizar seguimiento y evaluación permanente al mismo, con miras a ajustarlo o cambiarlo de acuerdo con las necesidades que se presenten, de tal forma, que los estudiantes logren:

- Integrar los conocimientos de formación adquiridos en el proceso académico con las realidades sociales.

- Participar activamente en los procesos de transformación educativa.
- Poner en juego las habilidades de investigación formativa para plantear proyectos pedagógicos acordes con las necesidades de la población y del medio en el cual se desenvuelven.

El proceso se ha ido cualificando de manera gradual mediante la contratación de asesores con formación y experiencia en investigación y con los niveles de exigencia en cada nivel de práctica, además de los requerimientos escriturales que obligan a las personas involucradas a reflexionar sobre sus prácticas, de manera intencionada, organizada y lógica, lo que trae como consecuencia docentes en formación más contextualizados. 


\section{Bibliografía}

Imbernon, F. (1996).La formación del profesor. Formar para innovar. Editorial Magisterio del Río de la Plata. Buenos Aires

Ministerio de Educación Nacional. (1996). Aportes sobre formación de Educadores. Resultados de los precongresos. (Documento de Trabajo)

Moreno Bayardo, M. G. (2002). Formación para la Investigación centrada en el desarrollo de habilidades. México: Universidad de Guadalajara.

Moreno, M. G. (2005): Potenciar la educación. Un currículum transversal de formación para la investigación”, en REICE, Revista Electrónica Iberoamericana sobre Calidad, Eficacia y Cambio en Educación, n.o 1, vol. 3, España, Recuperado en septiembre 23 de 2009, en http://www.ice.deusto.es/RINACE/reice/ Vol3n1_e/Res_Moreno.htm
Restrepo Gómez, B. (1999) Investigación en Educación. Bogotá, ICFES.

Restrepo Gómez, B. Conceptos y Aplicaciones de la Investigación Formativa, y Criterios para Evaluar la Investigación científica en sentido estricto. Recuperado el 23 de septiembre de 2009, de http://www.cna.gov.co/1741/ articles-186502 doc academico5.pdf

Schon, D. A. (1992). La formación de profesionales reflexivos. Hacia un nuevo diseño de la enseñanza y aprendizaje de las profesiones. Barcelona: Paidós.

Tecnológico de Antioquia (2008). Manual de prácticas interinstitucionales para las licenciaturas en educación básica y educación preescolar. En: www.tdea.edu.co

Tecnológico de Antioquia (2009). Proyecto Educativo Institucional. Documento en revisión.

UNESCO. (1998). Proyecto de declaración mundial sobre la educación superior en el siglo XXI: visión y acción. 